\title{
A tale of two centrifugal left ventricular assist devices
}

\author{
Jacob N. Schroder, MD, and Carmelo A. Milano, MD
}

From the Department of Surgery, Duke University Medical Center, Durham, NC.

Received for publication Feb 7, 2017; revisions received March 22, 2017; accepted for publication April 11, 2017; available ahead of print May 26, 2017.

Address for reprints: Carmelo A. Milano, MD, Professor of Surgery, Duke University Medical Center, Box 3043, Durham, NC 27710 (E-mail: carmelo.milano@duke.edu).

J Thorac Cardiovasc Surg 2017;154:850-2

$0022-5223 / \$ 36.00$

Copyright (c) 2017 Published by Elsevier Inc. on behalf of The American Association for Thoracic Surgery http://dx.doi.org/10.1016/j.jtcvs.2017.04.033

For almost a decade, the HeartMate II device (Abbott, Chicago, Ill) has been the most commonly installed durable left ventricular assist device (LVAD) in the United States, both as a bridge to transplant and for destination indications. This pump uses an Archimedes screw design with a resultant axial blood flow pattern; a single, central ruby bearing supports the rotor. Most recently, two publications have reported prospective, randomized trials in which the HeartMate II LVAD was compared with novel pumps that are smaller and use a centrifugal blood flow pattern, the HeartWare LVAD (HVAD; Medtronic, Minneapolis, Minn) and the HeartMate III (Abbott) LVAD ${ }^{1,2}$ (Figure 1). Although positive results with the HeartMate II device were responsible for the expansion of destination therapy, reports within the last 5 years have described the important issue of pump thrombosis, which frequently requires reoperation for device replacement. ${ }^{3,4}$ The rate of pump thrombosis with need for device replacement was reported as high as $10 \%$ during the first year after implantation. ${ }^{4}$ In addition, power cord wear and failure with the HeartMate II device was identified as another cause for pump stoppage and need for replacement. An important objective for these newer devices was therefore improvement in adverse event profiles relative to the HeartMate II, and reduction of pump thrombosis in particular.

The ENDURANCE trial (A Clinical Trial to Evaluate the HeartWare Ventricular Assist System) randomly allocated patients who were older and deemed ineligible for transplant to receive either the HVAD or the HeartMate II LVAD (2:1 randomization). ${ }^{1}$ The HVAD is a considerably smaller centrifugal pump with a magnetically levitated rotor; the pump is positioned entirely within the pericardium. The HVAD is already approved by the Food and Drug Administration for bridge to transplant indications, and the ENDURANCE trial sought to examine its performance during more chronic support as destination therapy. The primary end point was a composite consisting of survival free from disabling stroke or need for device replacement at 2 years. Therapy with the HVAD was equivalent to that with the HeartMate II with regard to achieving this primary end point $(55.4 \%$ vs $59.1 \%$ success, respectively). Furthermore, overall survival and

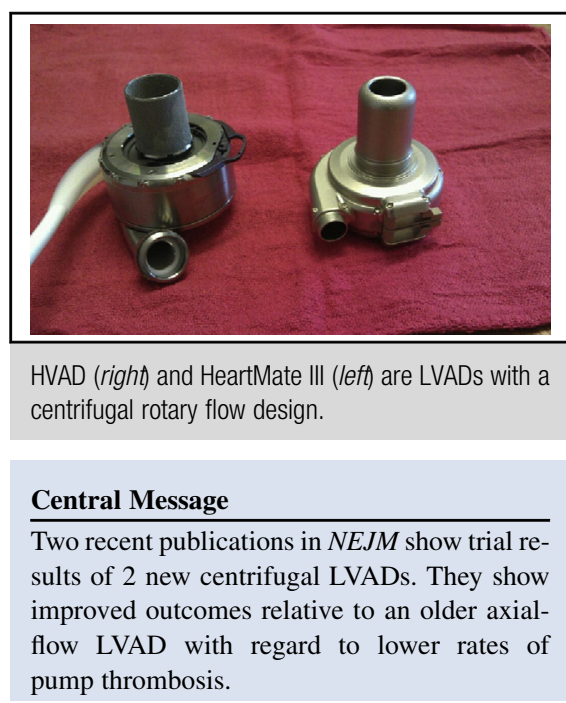

See Editorial Commentary page 853 .

freedom from disabling stroke were equivalent for the groups, and the HVAD group had fewer device failures relative to HeartMate II, mainly as a result of less pump thrombosis necessitating replacement in the HVAD group. Unfortunately, the benefit of reduced pump thrombosis for the HVAD was nullified by an increased overall stroke rate, which was roughly 3 times greater for the HVAD versus the HeartMate II (0.29 events/patient-y of support vs 0.09 events/patient-y of support, respectively). An increased stroke rate was previously reported in the ADVANCE bridge to transplant (Evaluation of the HeartWare Left Ventricular Assist Device for the Treatment of Advanced Heart Failure) trial, but this became more apparent in the ENDURANCE trial, probably because older patients were examined for a longer period of follow-up. ${ }^{1,5}$ Retrospective analyses of the ENDURANCE and ADVANCE data have shown an important association between hypertension during HVAD support and increased incidence of stroke. This has led to the ENDURANCE supplemental trial, in which destinationtherapy patients are again randomly allocated to HVAD versus HeartMate II, but those with HVAD placement are managed with increased blood pressure monitoring and a goal mean arterial blood pressure below $90 \mathrm{~mm} \mathrm{Hg}$. In this trial, patients were discharged with special equipment to monitor blood pressure, and they were required to maintain a log of daily measurements. The primary end point for this trial is neurological injury at 1 year, and the results should be submitted for publication this year. In addition, 


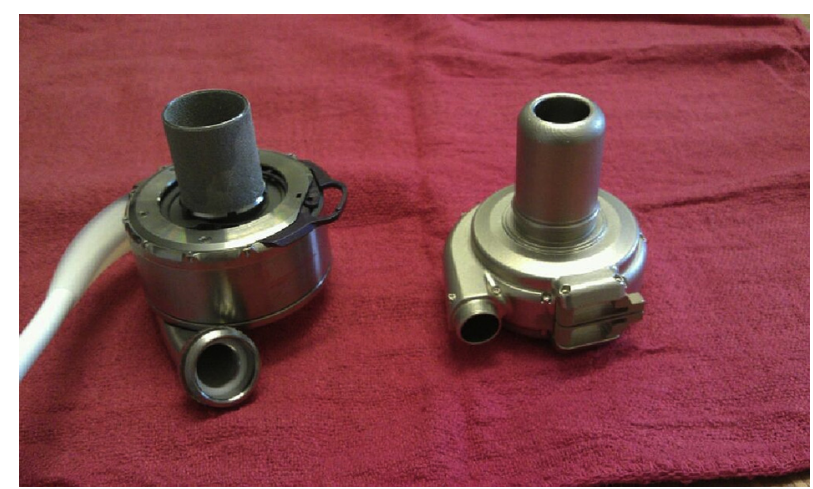

FIGURE 1. The HeartWare HVAD (right; HeartWare International Inc, Framingham, Mass) and the HeartMate III (left; Thoratec Corporation, Pleasanton, Calif) are left ventricular assist devices with a centrifugal rotary flow design. Use of a magnetically levitated rotor for both avoids bearings and improves durability.

many of us have observed thrombus formation in the left ventricle at the HVAD apical cannula and speculate this may be a source for both stroke and pump thrombosis with this device; redesign of this element might also affect stroke rates.

Whereas ENDURANCE showed equivalent outcomes with regard to the primary end point for HVAD versus HeartMate II, it should be stated that the smaller size affords the HVAD inherent advantages. Unlike the HeartMate II, the HVAD is placed entirely intrapericardially and does not require dissection of an upper abdominal pocket, resulting in shorter implantation times. The HVAD has been efficiently installed through a left anterior thoracotomy surgical approach, which may be advantageous for patients with multiple previous sternotomies or other unusual anatomy. A completed trial seeks to examine potential benefits for the thoracotomy approach, and results will be reported this year. All parts of the HVAD are smaller than in the HeartMate II, including the pump itself, the power cord, and the outflow graft, making it more suitable for pediatric and small adult applications. Application of the HVAD for pediatric patients is particularly exciting, because this population has not had a suitable, implantable system. In addition, the HVAD size and design have enabled use of the device for right-sided support; several reports have described patients who were successfully bridged to a heart transplant with 2 HVAD devices for right and left heart replacement. ${ }^{6}$ In essence, the HVAD provides surgeons an important degree of versatility that was not available with the HeartMate II LVAD.

In the same issue of The New England Journal of Medicine, the MOMENTUM 3 (Multi-center Study of MagLev Technology in Patients Undergoing MCS Therapy With HeartMate 3 IDE) trial is reported, in which patients were randomly allocated to receive the HeartMate III device versus the HeartMate II. ${ }^{2}$ The HeartMate III is also a blood pump with a centrifugal design and a completely magnetically levitated rotor. Like the HVAD pump, the HeartMate III has no bearings, which should achieve enhanced durability. Other important, unique design features for the HeartMate III include increased gaps (distances between the rotor and housing) and an algorithm for pump speed changes every 2 seconds to enhance washing of the pump. Finally, a modular power cord design facilitates cord replacement. Importantly, this report describes results from a short-term cohort followed up for only 6 months that included patients for whom the intention was destination or bridge to transplant. This report reviews outcomes from a very small subset of the entire trial (the larger trial includes 1000 patients who will be followed up for 2 years). The current report therefore represents a limited and early snapshot of the HeartMate III's clinical performance, and it is therefore premature to draw comparisons with the HVAD trial (ENDURANCE) in which an older cohort was followed for a substantially longer period.

The primary end point for MOMENTUM 3 is the same composite used in the ENDURANCE trial (freedom from death and disabling stroke on the original device). Patients treated with the HeartMate III LVAD more commonly achieved the primary end point than did those who received the HeartMate II at the 6 -month end point $(86.2 \%$ vs $76.8 \%$, respectively; $P=.037$ ). The mortality and rate of disabling stroke were similar for the groups, but there was a substantial reduction in the need for device replacement for the group who received the HeartMate III. In fact, there were no pump thromboses requiring replacement for the HeartMate III, whereas the HeartMate II continued to display a disturbing rate of replacement for pump thrombosis (11 replacements in 142 cases during the 6-month follow up). This would suggest that the new design features may have resulted in improved washing of the HeartMate III pump, preventing clot formation or deposition and subsequent hemolysis. Importantly, other adverse events, such as overall stroke and bleeding events, were similar between the groups (Table 1).

Although this MOMENTUM 3 cohort has only been followed up for a short duration (6 months), the problem of pump thrombosis appears to be positively affected. If so, this is an important milestone, because this event has been shown to affect morbidity, rehospitalization, and mortality. ${ }^{4}$ Furthermore, many speculate that pump thrombosis rates

TABLE 1. Rate of adverse events during 6-month follow-up in the MOMENTUM 3 trial

\begin{tabular}{lcc}
\hline \multicolumn{1}{c}{ Adverse Event } & HeartMate III & HeartMate II \\
\hline Stroke & $7.9 \%$ & $10.9 \%$ \\
Bleeding & $33.1 \%$ & $39 \%$ \\
Suspected pump thrombosis & $0 \%$ & $10.1 \%$ \\
\hline
\end{tabular}

Data represent percentage of cohort with adverse events. Adapted from Mehra and colleagues. $^{2}$ 


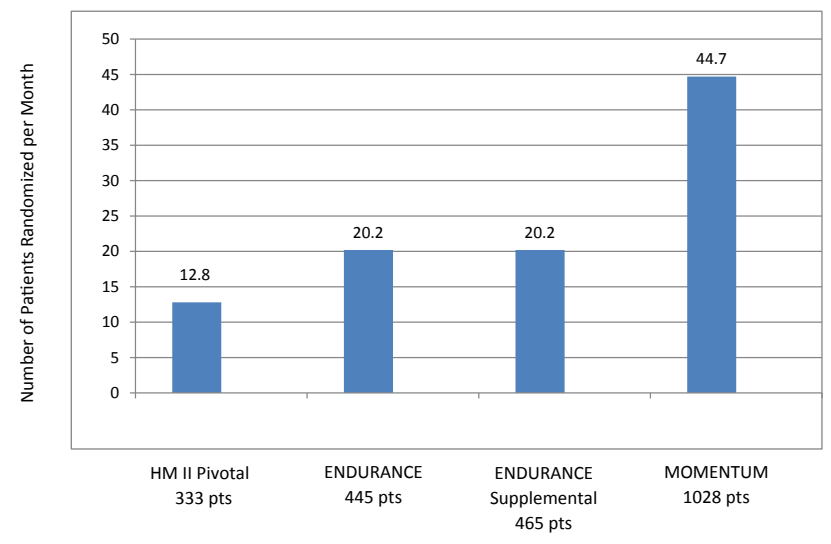

FIGURE 2. Rates of enrollment in randomized left ventricular assist device trials that have randomly allocated patients during the last 10 years. Total number of patients undergoing randomization has increased. Rate of enrollment has also increased. HM, HeartMate. (Data from Jacoski M, Medtronic; personal communication. Farrar D, St Jude Medical; personal communication, February 2, 2017).

with the Heartmate II device prevented the launch of the REVIVE-IT (Randomized Evaluation of VAD Intervention before Inotropic Therapy) trial to examine outcomes from LVAD support for a less sick cohort of patients with heart failure. In other words, the rates of device-related adverse events with the HeartMate II prevented its application to less sick patients who were not at high risk for death from heart failure. LVAD therapy in general will be advanced if the issue of pump thrombosis is truly eliminated with this newer design. More generally, it is encouraging that device design focused on this specific adverse event appears to have had a positive effect on clinical outcomes, giving hope that other difficult complications, such as devicespecific infection, bleeding events, and stroke, can be overcome with design change.

Another important and more general observation from these trials relates to the rate of enrollment and the time required to complete enrollment. For both these trials, a large number of patients were enrolled and randomly allocated in a relatively short period (MOMENTUM 3, $\mathrm{n}=1028$ patients; ENDURANCE, $\mathrm{n}=445$ patients). This represents a considerable change relative to earlier device trials, such as the REMATCH (Randomized Evaluation of Mechanical Assistance for the Treatment of Congestive Heart Failure) trial or even the HeartMate II destination trial, which enrolled patients much more slowly. The rapid completion of enrollment for these trials results from the overall increased clinical application of the technology, effective trial design, and an engaged research community (Figure 2). The rapid enrollment portends well for the future investigation of newer LVAD designs, testing of modification of existing devices, or trials of novel LVAD management strategies. The faster that we can test and answer questions in this field, the more effective the therapy will be for patients with advanced heart failure.

\section{Conflict of Interest Statement}

C.A.M. is a consultant and educator for Medtronic, Minneapolis, Minn, and for Abbott, Chicago, Ill. J.N.S. has nothing to disclose with regard to commercial support.

\section{References}

1. Rogers JG, Pagani FD, Tatooles AJ, Bhat G, Slaughter MS, Birks EJ, et al. Intrapericardial left ventricular assist device for advanced heart failure. $N$ Engl J Med. 2017;376:451-60.

2. Mehra MR, Naka Y, Uriel N, Goldstein DJ, Cleveland JC Jr, Colombo PC, et al; MOMENTUM 3 Investigators. A fully magnetically levitated circulatory pump for advanced heart failure. N Engl J Med. 2017;376:440-50.

3. Slaughter MS, Rogers JG, Milano CA, Russell SD, Conte JV, Feldman D, et al; HeartMate II Investigators. Advanced heart failure treated with continuous-flow left ventricular assist device. N Engl J Med. 2009;361:2241-51.

4. Starling RC, Moazami N, Silvestry SC, Ewald G, Rogers JG, Milano CA, et al. Unexpected abrupt increase in left ventricular assist device thrombosis. $N$ Engl J Med. 2014;370:33-40.

5. Aaronson KD, Slaughter MS, Miller LW, McGee EC, Cotts WG, Acker MA, et al; HeartWare Ventricular Assist Device (HVAD) Bridge to Transplant ADVANCE Trial Investigators. Use of intrapericardial, continuous-flow, centrifugal pump in patients awaiting heart transplantation. Circulation. 2012;125:3191-200.

6. Milano CA, Schroder JN, Daneshmand MA. Total artificial heart replacement with 2 centrifugal blood pumps. Oper Tech Thorac Cardiovasc Surg. 2016;20:306-21. 